\title{
Una revisión sistemática sobre las relaciones entre el guanxi y el capital social en el contexto chino
}

\section{A systematic review about the relationships between guanxi and social capital in the Chinese context}

\author{
Haowen $\mathrm{Li}$ \\ $\mathrm{UAB}$ \\ lijoven0919@gmail.com \\ Xavier Úcar \\ $\mathrm{UAB}$ \\ xavier.ucar@uab.cat
}

\begin{abstract}
Resumen: El guanxi se confunde fácilmente con el capital social. Muchos estudios sobre ambos fenómenos han aplicado en China los conceptos, métodos y teorías de la sociología occidental ignorando la importancia de las influencias socioculturales de las culturas respectivas. Este artículo realiza un análisis sistemático de las investigaciones realizadas sobre el guanxi y el capital social en la última década. Se analizan los resultados de 39 artículos seleccionados a través del método PRISMA. De dicho análisis se desprenden los límites y contradicciones existentes en relación con el guanxi tanto desde la perspectiva del confucianismo como de la del propio concepto occidental de capital social. Se desvela, también, el significado esencial del guanxi en la sociedad china y las relaciones que puede establecer con el capital social, dado que, aunque son dos conceptos diferentes, pueden conectarse e influirse recíprocamente. Por último, se sintetizan las conclusiones del estudio.
\end{abstract}

Palabras clave: guanxi, capital social, confucianismo, relación social, renqing, intercambio.

Abstract: Guanxi are easily confused with Social Capital. The concepts, methods and theories of western sociology have been widely applied to the study of these two phenomena in China, but the influence of social culture on them have been ignored. This paper made a systematic analysis of the researches on Guanxi and Social Capital in the last decade and analyzed the results of selected 39 articles 
by PRISMA. The analysis indicated the limits and contradictions of Guanxi from the perspectives of Confucianism and the concept of western social capital, revealed the essential meaning of Guanxi in Chinese society. Besides, it can be seen that although Guanxi and Social Capital are two different concepts, they have a mutual influence. Finally, the conclusions of the study are synthesized.

Keywords: guanxi, social capital, Confucianism, social relationship, renqing, exchange. 


\section{INTRODUCCIÓN}

En la transición económica hacia los sistemas de mercado, producida en China a lo largo de las tres últimas décadas, existe una aparente paradoja. Por un lado, el milagro económico podría atribuirse en parte a la existencia de un importante capital social en China, pero, por otro, aparecen numerosos problemas relacionados con el subdesarrollo y la disfunción organizacional (Peck y Zhang, 2013). En la sociedad tradicional china, era la familia la que proporcionaba la confianza básica que posibilitaba el establecimiento de relaciones sociales. Sin embargo, en la sociedad contemporánea, las interacciones entre las personas van más allá de la familia y empieza a aparecer gradualmente lo que se podría caracterizar como una «sociedad de desconocidos». En esta nueva sociedad el capital social tradicional no constituye, en principio, una ventaja, y eso ha supuesto una crisis de confianza que está obstaculizando en gran medida el desarrollo económico chino.

Algunas investigaciones afirman que el guanxi es una forma de capital social, constituido por recursos que se derivan de las relaciones interpersonales (Qi, 2013; Sennett, 2012). Muchos estudios sobre guanxi y capital social han aplicado en China los conceptos, métodos y teorías de la sociología occidental. Eso los ha llevado, a menudo, a caracterizar el guanxi como una forma de capital social, fundamentalmente porque se genera a partir de relaciones de confianza. También a aplicar, de manera indiscriminada en aquel contexto, la teoría occidental elaborada sobre el capital social. Sin embargo, una tal aplicación supone ignorar las diferencias culturales existentes entre ambos sistemas socioculturales. Por eso resulta necesario aclarar, desde la perspectiva cultural china, algunos conceptos básicos sobre el guanxi y, asimismo, los requisitos previos para que aquel pueda hacerse efectivo. En este marco planteamos las siguientes preguntas de investigación: ¿cuál es el significado esencial del guanxi en la sociedad china? y ¿qué tipo de relación existe entre el guanxi y el capital social?

Para responder a estas cuestiones analizamos las investigaciones desarrolladas en la última década en relación con el guanxi y el social capital.

En el contexto de la actual modernización, el desarrollo económico y social de China tendría que basarse en la agregación, reformulación e integración no solo del capital social tradicional, sino, también, de nuevos tipos de capital social derivados de las relaciones de dicho país con las sociedades de Occidente. Desde nuestro punto de vista, la investigación sobre el capital social puede aportar conocimientos y estrategias que ayuden al desarrollo de la sociedad civil china; a la comprensión de las problemáticas generadas por la transición de la sociedad agrícola tradicional a la sociedad industrial y tecnológica moderna; y, asimismo, a brindar un apoyo teórico para el desarrollo estable de la economía. Es con esta idea que hemos organizado el trabajo en tres apartados en los 
que presentamos, en primer lugar, la metodología del análisis sistemático. A continuación, los resultados obtenidos sobre el guanxi, las formas como se construye y las relaciones que mantiene con el capital social. Acabamos, por último, con algunas conclusiones derivadas de los análisis conceptuales desarrollados.

\section{METODOLOGÍA}

Antes de definir las palabras claves para iniciar el análisis sistemático se realiza una revisión exploratoria a través del buscador genérico Google Scholar. Tras obtener una panorámica general y definir las palabras claves, se consultan en tres bases de datos en inglés y en chino: Erik, Cnki.net y Wiley online library. Cnki.net es la base de datos más grande existente en China y ha sido diseñada con todo el Sistema Integrado de Recursos de Conocimiento de aquel país. La cantidad de artículos encontrados en las bases de datos es pequeña y buena parte de la investigación sobre la temática se ha producido sobre todo en la última década. Es por eso por lo que tomamos este periodo, 2011-2020, como criterio de análisis para la revisión de la literatura académica y la selección de artículos. La Tabla 1 muestra las palabras claves, sus combinaciones en las búsquedas y los resultados obtenidos.

TABLA 1

Combinación de palabras claves, bases de datos y criterios de inclusión

\begin{tabular}{ccccc}
$\begin{array}{c}\text { Combinación de términos } \\
\text { buscados }\end{array}$ & ERIC & $\begin{array}{c}\text { Cnki.net } \\
\text { (en chino) }\end{array}$ & $\begin{array}{c}\text { Wiley } \\
\text { online } \\
\text { library }\end{array}$ & $\begin{array}{c}\text { Criterios de inclusión } \\
\text { y fecha de búsqueda }\end{array}$ \\
\hline inglés y chino) & &
\end{tabular}

Chinese social capital AND guanxi

$\begin{array}{llll}\text { AND Confucianism AND } & 32 & 6 & 57\end{array}$

Generation mechanism

Guanxi AND Chinese social De 2011 hasta 2020 capital AND Market economy AND confidence AND Generation $18 \quad 75 \quad 2$ mechanism Fecha de búsqueda: 19 de enero de 2021

Renqing AND Chinese capital social AND social relation AND $\begin{array}{lll}71 & 16 & 24\end{array}$

Generation mechanism

\begin{tabular}{lllll} 
TOTAL referencias: & 121 & 97 & 83 & 301 \\
\hline
\end{tabular}


Para el proceso de análisis de los resultados de la búsqueda bibliográfica se utiliza el diagrama de flujos PRISMA (Moher et al., 2009), que caracteriza en tres pasos los criterios de exclusión y la selección final de los artículos.

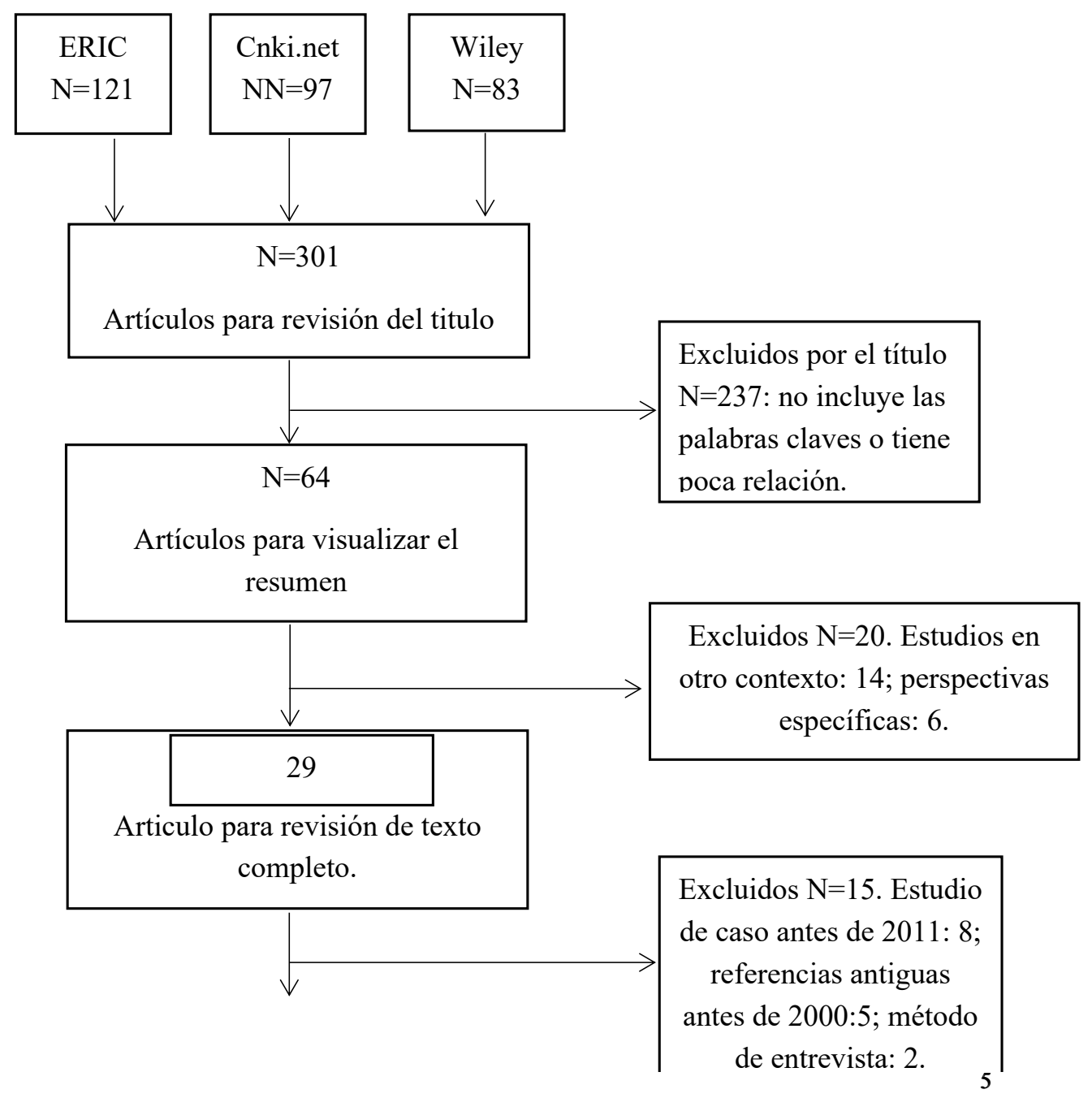

Siguiendo dicha metodología se revisan, en un primer paso, los títulos de los 301 artículos encontrados y se excluyen aquellos que no usan las palabras claves o que tienen poca relación con el objeto de estudio. En el segundo paso, después de revisar sus resúmenes, se excluyen 20 textos. La exclusión obedece, por un lado, al hecho de que la 
mayoría de los artículos se refieren a perspectivas demasiado específicas que ponen en relación el capital social con, entre muchos otros, la gimnasia, la vida rural, la relación médico-paciente (Zou, Cheng y Nie, 2018), y el trabajo de las mujeres. Zhang (2011) examina, por ejemplo, cómo el guanxi influye en las relaciones laborales de las mujeres migrantes rurales. Por otro lado, algunas investigaciones encontradas son de países que no tienen que ver con la cultura china, por lo que también se decide excluirlos. Por ejemplo, Vélez-Calle, Robledo-Ardila y Rodríguez-Ríos (2015) estudian el fenómeno guanxi en la cultura de América Latina y lo hacen equivalente a lo que denominan «compadrazgo». Cabe destacar que se mantienen los artículos relacionados con la presencia de inmigrantes chinos en otros países para poder analizar la influencia del guanxi en diferentes grupos chinos. En el último paso se revisan 44 textos completos. De ellos se acaba eliminando 15. En unos casos porque, a pesar de haber sido publicados en los últimos años, el estudio de caso presentado y los artículos citados eran muy antiguos y poco relevantes. La realización de una única entrevista a fin de analizar la opinión de un informante sobre la función del guanxi y el social capital. El resultado final corresponde a 29 textos que resultan relevantes para el análisis sistemático. Durante la lectura de los textos aparecen referencias citadas frecuentemente por lo que se realizan búsquedas complementarias con el propósito de de complementar el análisis previo. Se han agregado 10 textos más, por lo que el análisis de contenido se realiza, finalmente, sobre 39 artículos.

\section{RESULTADOS DEL ANÁLISIS SISTEMÁTICO}

El inicio del interés académico en el guanxi puede datarse en la última década del siglo xx. En aquellos años se produjo una creciente concentración en el guanxi como un campo explícito de investigación y estudio. Se trataba de conocer la incidencia, la forma y la importancia del guanxi, en tanto que tipo de relación o conexión típica entre personas en las áreas culturales chinas. La discusión sobre el guanxi se centra, principalmente, en el papel que juega en los negocios; algo que refleja la atención de los investigadores occidentales hacia el resurgimiento de China en la década de 1980 y las relaciones características propias de su economía. El papel fundamental del guanxi en los negocios chinos es indiscutible, pero el guanxi no se limita a las relaciones comerciales, sino que también es esencial para las relaciones políticas y sociales. De hecho, esto no solo se da en China, sino también en Taiwán, Hong Kong, Singapur y, asimismo, entre las poblaciones chinas de otros países. El guanxi ha sido descrito como un concepto sociocultural fundamental para la comprensión de la estructura social china. No hay duda de que el 
guanxi es un componente importante de la estructura social tradicional, pero es también muy significativo en la China del siglo xxi.

El guanxi es un elemento fundamental para comprender la cultura china y las relaciones entre las personas, los grupos y las organizaciones que se producen en su seno. Originalmente, el guanxi se refiere al vínculo de parentesco y de la familia política. También a vínculos generados a través de relaciones estables con otras personas que se sostienen sobre el intercambio de favores (renqing) y una serie de obligaciones recíprocas. Pero una definición así se queda muy corta dada la riqueza de connotaciones que acompañan a este término en la cultura china.

El establecimiento, desarrollo, mantenimiento o reconstrucción del guanxi requiere una inversión considerable de tiempo y de recursos por parte de las personas. Especialmente en algunos de los momentos y ocasiones en que la cultura china considera más importantes, como los festivales tradicionales, las bodas, las fiestas familiares y de cumpleaños, etc. Todos estos eventos se constituyen en oportunidades para las personas y las familias para construir y mantener el guanxi. En síntesis, la cultura guanxi supone que las personas están obligadas a proporcionar favores a sus familiares y a aquellas personas con las que mantienen este tipo de relación y, también, a esperar favores mutuos en el futuro.

A través de la revisión de los artículos académicos seleccionados se ha encontrado que la mayoría de ellos se refiere a que el guanxi es un capital social que proviene de la influencia de los valores confucianos y del desarrollo de la parentalidad y las relaciones vinculadas al territorio en la sociedad china. A continuación se presentan las diferentes perspectivas de los autores respecto el guanxi y el social capital.

\section{Diferentes perspectivas sobre el guanxi}

La investigación sobre el guanxi se da principalmente en las disciplinas de antropología, estudios de negocios, psicología social y cultural y sociología. Hay referencias cruzadas sobre el guanxi entre estas diferentes disciplinas. A pesar de eso, la gran mayoría de los estudios sobre el guanxi se publican en revistas de negocios, gestión y marketing (Liu y Mei, 2015; Qi, 2012). En los artículos seleccionados identificamos tres perspectivas sobre el guanxi: ego-práctica, ética comunitaria y relación confuciana. Las bases teóricas y las evaluaciones del guanxi son diferentes desde cada perspectiva.

La perspectiva ego-práctica es la dominante en la investigación del guanxi en el campo sociológico. Según la teoría del capital social, los individuos forman parte de redes sociales. Las redes de guanxi son consideradas como recursos sociales que las 
personas desarrollan y despliegan para obtener favores y beneficios; como ascenso laboral y éxito profesional (Luo et al., 2012). La investigación del guanxi individual e interpersonal se basa principalmente en las teorías del intercambio social y, específicamente, en el intercambio entre líderes y subordinados. Este último se refiere a las relaciones especiales que los líderes establecen con sus subordinados debido a la existencia del guanxi entre ellos. Eso significa que dichos subordinados obtienen un trato privilegiado en la empresa.

La naturaleza esencial de la interacción guanxi es el intercambio de recompensas y beneficios entre los participantes. La perspectiva ego-práctica tiende a centrarse en probar y documentar los efectos del guanxi en la mejora de la situación y posicionamiento de los individuos. Sin embargo, algunas investigaciones cuestionan los resultados positivos del guanxi en las organizaciones y en las transacciones comerciales. En primer lugar, a pesar de que el guanxi no se equipara con la corrupción y el amiguismo, Khatri, Tsang y Begley afirman que «tiene deficiencias corrosivas» (2006: 63). En segundo lugar, las transacciones de guanxi requieren un manejo hábil y estratégico de la administración de los recursos sociales y las jerarquías (Luo, 2019). En tercer lugar, y conectando ya con la siguiente perspectiva, se puede afirmar que, en contraste con la perspectiva ego-práctica, los estudios de la perspectiva ética comunitaria exploran, específicamente, los aspectos corrosivos del guanxi dentro de las organizaciones y la sociedad en general.

Los investigadores que adoptan la perspectiva de la ética comunitaria (Huang, Huang y Dubinsky, 2014; Luo, Huang y Wang 2012; Chen et al., 2011) plantean las influencias negativas inherentes a las prácticas del guanxi. Unas prácticas que impactan en los intereses colectivos de las organizaciones y la sociedad y, también, en el desarrollo y el funcionamiento de la moral pública. Aquellos autores plantean que las prácticas de guanxi son potencialmente poco éticas. Es decir, benefician solo a los individuos y, en concreto, tan solo a los privilegiados y violan, asimismo, las normas sociales locales de justicia y equidad. Chang (2011) argumenta, además, que las redes de guanxi en China se formaron en condiciones de escasez de recursos.

Desde la perspectiva relacional confuciana, el guanxi y el capital social parecen ser bastante diferentes en China respecto de las conceptualizaciones occidentales. Las características de las relaciones sociales chinas se manifiestan principalmente en la orientación al guanxi y en la omnipresencia de su práctica en la vida cotidiana. La centralidad del guanxi en China se basa en filosofías antiguas, especialmente en el confucianismo, que ha dominado la cultura y los sistemas de gobierno chino durante más de dos mil años.

El confucianismo sostiene que los seres humanos están fundamentalmente orientados al guanxi. La clave del orden social consiste en construir una jerarquía de relacio- 
nes fuertes y ordenadas (Ke, 2018). En la sociedad ideal definida por Confucio, el individuo es un ser relacional y la sociedad se forma sobre la base de redes personales y sociales. Los miembros de la sociedad son estrechamente interdependientes de los demás y están integrados en el contexto social. Eso significa, desde aquella perspectiva, que las relaciones entre diferentes individuos, sociedades y organizaciones son armoniosas. La ética del confucianismo también enseña a las personas a respetar a los patriarcas y a los ancianos; lo que significa que se brinda respeto a todo tipo de autoridad (Jia, You y Du, 2012). El confucianismo surgió en una sociedad rural donde las personas no viajaban lejos de su comunidad y rara vez contactaban con el mundo exterior, manteniendo aislado su propio círculo social o conocido. Las personas aplican «diferentes reglas de intercambio social cuando interactúan con personas en diferentes tipos de relaciones o diferentes grados de intimidad» (Confucio, 2013: 158). En otras palabras, tratan a cada persona de manera diferente en función de diversos criterios relacionados con el parentesco, la familiaridad y, en general, el grado de conocimiento y cercanía. Fei (2015) define la relación social china como un «patrón de ondas sucesivas». Esto le permite describir la estructura social china utilizando la imagen de una onda formada por una piedra arrojada a un estanque. Cada individuo está rodeado por una serie de círculos concéntricos producidos por la propia capacidad de influencia social. Cada red de relaciones sociales considera a cada yo como el centro. Cada círculo, a medida que se extiende y aleja del centro, se vuelve más distante y menos significativo. Hay una mayor confianza en el interior y menor confianza en el exterior de los círculos. Esto supone que se manifiestan diferentes actitudes hacia los seres más cercanos y hacia los extraños. Este concepto, patrón de ondas sucesivas, es muy popular en el campo de investigación del guanxi y el capital social. Como el guanxi juega un papel esencial en la obtención de recursos en la sociedad china, las personas lo consideran como un recurso y lo cultivan «energética, sutil e imaginativamente» (Sennett, 2012: 135).

\section{El guanxi y el renqing}

La noción de renqing, que se puede traducir como obligaciones y endeudamientos recíprocos, es fundamental en el sistema del guanxi en China, ya que hace que el guanxi sea algo más que una simple conexión o relación social. El renqing significa que existe una relación interpersonal, que se manifiesta en obligaciones sociales hacia el otro y en conductas mutuas de reciprocidad. En la sociedad china, las personas que carecen de 
renqing son excluidas de la vida social y de los intercambios e interacciones que aquella supone.

Se puede considerar que el guanxi es un sistema de intercambio de regalos y favores entre personas en el que se fabrica renqing y, como señala Qi (2013), en relación con la duración de las obligaciones derivadas, no hay un límite temporal para el reembolso. El intercambio mutuo, moralmente infundido, es llevado a cabo a través del renqing, que, según aquel mismo autor, es la fuerza dinámica detrás de la práctica del guanxi. Las relaciones con guanxi pueden cultivarse y, dado que implican reciprocidad, obligaciones, evaluaciones emocionales y compromisos morales, pueden ser entendidas como una forma de intercambio asimétrico de favores entre personas sobre la base de renqing (Barbalet, 2018). La calidad del intercambio de renqing y el grado de reciprocidad que surge de él van a determinar la solidez en el futuro de las obligaciones derivadas del guanxi.

\section{¿Cómo se construye el guanxi?}

Zhang, Deng y Wang (2014) sugieren que el guanxi chino a menudo se basa en experiencias y asociaciones sociales compartidas con familiares o personas de una misma clase social. El guanxi también implica conexiones sociales construidas implícitamente, sin necesidad de discusiones explícitas o arreglos sobre intereses y beneficios mutuos. Una vez que dos personas han establecido un nivel suficiente de guanxi, cada una puede solicitar un favor a la otra sabiendo que la oportunidad de corresponder surgirá en un momento $u$ otro en el futuro.

Por ejemplo, si la persona A, que tiene guanxi previo con la persona $\mathrm{C}$, necesita la ayuda o el favor de la persona $\mathrm{B}$, a la que no conoce, entonces A puede pedirle a $\mathrm{C}$, que tiene su propia relación de guanxi con $\mathrm{B}$, que le haga de intermediaria. A través de tales intercambios A se beneficia del guanxi que tiene C con B. De esta manera el guanxi de $\mathrm{C}$ con $\mathrm{B}$ se transfiere a $\mathrm{A}$. Como consecuencia de esta interacción, $\mathrm{A}$ debe renqing a $\mathrm{B}$ y C. Las relaciones recíprocas que ahora operan entre A, B y C significan que, si alguno de ellos falla, no solo se ve afectada la relación de una persona con otra persona en particular, sino también su relación con toda la red guanxi. Hay que señalar que, a veces, las personas que participan en redes de guanxi pueden sentirse como aprisionadas en estas telarañas de interrelaciones, débitos y obligaciones múltiples. Como hemos señalado, cumplir con la obligación recíproca requiere mucho tiempo y esfuerzo.

En la Figura 1 se pueden ver, de una manera gráfica, estas relaciones que involucran el guanxi y el renquing. 
FIGURA 1

Ejemplo de funcionamiento del guanxi y el renquing

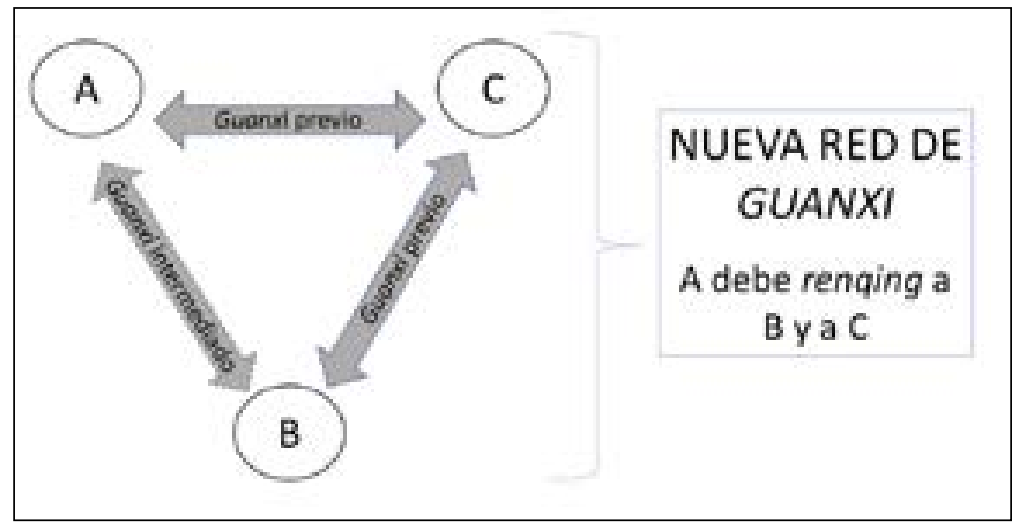

Se puede hablar de tres tipos de guanxi: el que se produce en una relación familiar; en una relación con personas conocidas; y, por último, en una relación con personas desconocidas.

Es a partir de la base de los lazos de sangre y el parentesco que los miembros de la familia forman la relación interna más cercana. Este tipo de guanxi implica que los miembros de la familia están comprometidos sin condiciones en el cuidado recíproco.

El segundo tipo de guanxi es el que se deriva de la relación con personas cercanas o conocidas. Esta red de guanxi puede estar integrada por parientes, amigos, compañeros de clase, vecinos, compañeros de misma universidad o de la tierra natal. Los parientes prestan más atención a las obligaciones morales, mientras que las amistades prestan más atención a la igualdad y la reciprocidad. El principio que rige la relación entre conocidos es la reciprocidad, es decir, el destinatario no puede olvidarse nunca de devolver un favor (renqing) previamente prestado. Si ignora la reciprocidad, afectará seriamente su reputación social.

El tercer tipo de guanxi se refiere a la relación con personas desconocidas. En tanto que personas que no forman parte de los círculos más cercanos y no están vinculadas por obligaciones recíprocas, suelen ser inicialmente, objeto de sospecha. Es decir, se presupone que solo se van a preocupar por las ganancias y pérdidas personales que se deriven de la relación. En las relaciones con extraños, la interacción suele ser, en general, superficial y temporal, dado que se presupone que el oportunismo va a ser la regla de la interacción.

Todas las personas están vinculas por la relación de guanxi, que impone ciertas responsabilidades y deberes. Esta red interdependiente funciona como una institución de 
seguridad informal que evita que los participantes la abandonen, cometan fraude, explotación de los otros o traición a su confianza. De ahí que se garantice la fidelidad de todos los participantes. Otros trabajos empíricos también muestran que el guanxi es fundamental para lograr el éxito empresarial en China (Li et al., 2011). Los teóricos señalan que el guanxi funciona como un sustituto de las protecciones legales, proporcionando «confianza contextual» para generar y sustentar la confianza entre las personas (Wang, 2014).

\section{Diferentes perspectivas entre Occidente y Oriente sobre el guanxi}

Existen diferentes opiniones sobre los efectos de las relaciones fuertes y débiles en las sociedades occidental y oriental. Granovetter (1983) enuncia la teoría de la «hipótesis de la relación débil», es decir, en la sociedad estadounidense sucede que cuantas más personas se conocen superficialmente en todos los ámbitos, más fácil es conseguir lo que se busca. Por el contrario, los investigadores chinos Yanjie y Lei (2013) presentan la «hipótesis de la relación fuerte». En la sociedad china lo importante no es la amplitud y diversidad de información obtenida a través de «relaciones débiles», sino la ayuda definitiva y poderosa que puede brindar una «relación fuerte», es decir, aquella proporcionada por los vínculos generados en la familia nuclear, con los amigos cercanos y con los socios comerciales. Quizás por eso Sennett (2012) considera que el término «deber» describe mejor que el de capital social esas relaciones fuertes. De hecho, la connotación de «red de relaciones» en china es mucho más compleja que en la sociedad occidental. El guanxi de la sociedad china a menudo es considerado como una «caja negra» en la que no se acaba de saber muy bien qué sucede entre el deseo de un individuo de conseguir una meta y el logro final de la misma. Dicho proceso se realiza a través del guanxi de formas que, a menudo, resultan muy poco transparentes.

En una economía globalizada, caracterizada por un rápido avance tecnológico y una alta incertidumbre sociopolítica, se requiere la fuerza tanto del sistema oriental del guanxi como del sistema de mercado occidental. Se podría decir, siguiendo a Wang y Kang (2012), que la sociedad occidental confía demasiado en las reglas impersonales y universales, y se centra en transacciones discretas a corto plazo, mientras que en Oriente a veces se confía demasiado en el apego y la benevolencia del grupo interno, descuidando la capacidad y la integridad de las personas. Mao, Peng y Wong (2012) ejemplifican esto señalando que utilizar las relaciones personales para influir en una entrevista de trabajo en favor de un familiar o un amigo es una práctica más aceptada en China que en las sociedades occidentales. Estos mismos autores apuntan que si en China se rechaza ofrecer dicha ayuda, la persona que hace es considerada apática o, incluso, poco ética. 


\section{El capital social}

Algunos sostienen que el guanxi es una forma especial o una variante del capital social (Qi, 2013; Xiaoxin, 2013). De una manera simplificada se podría decir que la idea central de la teoría del capital social es que las relaciones sociales se constituyen como un recurso esencial para la emergencia y el sostenimiento de las acciones y proyectos colectivos. Siguiendo a Bourdieu (1986), el capital social sería el total de recursos reales o potenciales ligados a la posesión de una red duradera de relaciones de conocimiento y reconocimiento mutuo. En la mayoría de las investigaciones las definiciones de referencia sobre el capital social son las de Putnam (1993), Coleman (1988) y Bourdieu (1986). Coleman (1988) enfatiza la naturaleza estructural del capital social y las características que lo definen a partir de las relaciones interpersonales que se producen en el marco de las estructuras sociales. Según este autor, el capital social es resultado de las relaciones interpersonales y está formado por el conjunto de elementos de la estructura social que facilitan a las personas emprender acciones colectivas. Las normas y sanciones, por ejemplo, pueden disminuir la criminalidad y permiten a las instituciones generar estabilidad en contextos de inestabilidad. Putnam (1993), por su parte, señala que el capital social emerge como resultado de la cooperación organizada entre los miembros de la comunidad que actúan y establecen relaciones a partir de la interiorización de determinados valores como la confianza, la solidaridad y la reciprocidad. También a partir de la existencia del tejido social y de las normas e instituciones que lo configuran. Define, por otra parte, tres tipos de capital social que podrían relacionarse, de alguna manera, con la idea de Fei (2015) del patrón de ondas sucesivo y con los tres tipos de guanxi caracterizados: el familiar, el de las personas conocidas y el de los desconocidos. Putnam (1995) se refiere al capital social tipo «bonding», que se genera a partir de vínculos intragrupo regulados por lazos de exclusividad/exclusión. Suelen ser vínculos fuertes y se producen en el marco de grupos homogéneos como los amigos y la familia. El tipo «bridging», que se refiere a vínculos con personas conocidas, asociadas o colegas lejanos. Son lazos de inclusión, que buscan incluir a los otros en «lo propio». Y, por último, el tipo «linking», que se refiere a las relaciones que se establecen con individuos y grupos con diferentes estatus y poder. Es la capacidad de conseguir recursos, ideas e información de instituciones formales más allá de la propia comunidad de referencia (Woolcock, 2001).

Todas estas definiciones coinciden en caracterizar el capital social como un conjunto de recursos disponibles para los individuos que son adquiridos a través de su participación en redes sociales. En función de los diferentes autores se enfatizan diferentes elementos como la confianza, la reciprocidad, la cooperación, las normas (compartidas, de reciprocidad, etc.), los valores, las instituciones, etc. (Padrò y Úcar, 2015). 
Tres son las dimensiones esenciales para entender el capital social: la estructural, la relacional y la orientada a los recursos. La primera perspectiva enfatiza las características (densidad, amplitud, etc.) de la estructura que configura la red relacional generadora de capital social. En las unidades básicas de cada red las relaciones se generan y mantienen sobre la confianza y la reciprocidad entre dichas unidades. La segunda enfatiza el valor de los recursos sociales generados, esto es, las redes sociales que se forman, su estabilidad y durabilidad, a partir de relaciones que pueden ser entre individuos o grupos. La tercera perspectiva, por último, enfatiza el acceso a los recursos y considera el capital social como la capacidad para conseguir beneficios en virtud de la pertenencia a redes sociales. Esta perspectiva parece ser la más próxima o, al menos, la que ofrece una mejor definición del nivel individual de capital social en un contexto guanxi.

\section{El capital social chino}

Como se ha comentado, el capital social tradicional chino se basa, en primer lugar, en la ética confuciana y en las normas morales como orientación básica de valor. En segundo lugar, hemos apuntado que se construye sobre relaciones de consanguinidad y pertenencia territorial. Las organizaciones de clanes basadas en consanguinidad brindan a los miembros de la familia funciones que, hoy en día, son llevadas a cabo principalmente por las organizaciones sociales modernas (Zheng, 2011). En la sociedad tradicional, la familia es una entidad social con funciones integrales, que incluye, entre otras, funciones políticas, legales, económicas, religiosas, educativas y de ayuda mutua. Con el desarrollo de la división social del trabajo y la aparición de clases, las relaciones y las actividades sociales se vuelven cada vez más complejas. Las organizaciones sociales se forman y funcionan cada vez más para satisfacer las necesidades de sus miembros; normalmente grupos de personas que buscan beneficios comunes. Con la expansión del alcance geográfico de la producción y la vida, las organizaciones sociales basadas en relaciones geográficas y de clase compensan en cierta medida la función de las organizaciones de clanes tradicionales y desempeñan un papel social más importante en las ciudades, convirtiéndose en una estructura informal fundamental de cooperación social.

En tercer lugar, el capital social tradicional chino es relativamente cerrado (Wang y Kang 2012). La relación de consanguinidad de una familia siempre está limitada a los miembros que la configuran, cosa que dificulta relaciones con otras familias. Es una relación cerrada que dificulta la comunicación externa. Por otro lado, en la sociedad agrícola, las personas vivían en un área territorial determinada fija; las áreas eran relativamente independientes y la movilidad no era ni frecuente ni demasiado conveniente. 
Con el desarrollo de la economía de mercado, se requiere que la red de relaciones sociales se desarrolle a través del parentesco y entre diferentes regiones para poder formar una amplia gama de relaciones de comunicación y permitir una mejor asignación de los recursos sociales (Sang, 2011). En este caso, la cercanía de la red relacional de la sociedad tradicional tiende a debilitarse para adaptarse al desarrollo de la economía de mercado.

El capital social generado por esta relación sigue basándose en el parentesco: cuanto más cercano, el capital social es más fuerte. Eso significa que las actividades de un individuo, fuera de su red de referencia, están muy restringidas. Por otra parte, la naturaleza de las relaciones propias del capital social, en sentido occidental, requieren que las personas realicen intercambios a nivel horizontal en el marco de la sociedad civil; unos intercambios que no son obligatorios, sino que se basan en la voluntariedad y el consenso (Li, 2013). Este intercambio amplio y libre basado en la voluntad de las personas puede contribuir a romper la interdependencia mutua del parentesco y a promover, más allá de las constricciones de guanxi, la formación de unos modos de cooperación espontáneos en la sociedad civil.

En el marco sociocultural chino el desarrollo del individuo toma la relación de consanguinidad y proximidad geográfica como el camino a seguir. La relación social establecida por el individuo es modulada por estos dos aspectos, en lugar de desarrollarse en la dirección horizontal a la que nos hemos referido, lo que determina que la generación de capital social sea muy lenta. No obstante, una vez que se forma dicho social capital, es difícil cambiarlo y renovarlo.

La sociedad actual china se caracteriza, en general, por una baja confianza entre las personas. Se rige por el denominado «familiarismo» (familialism) (Ip, 2008; Wijaya, 2008), que supone una clara orientación al mantenimiento de la gran mayoría de asuntos dentro del entorno familiar y al establecimiento generalizado de normas sociales uniformes para todas las personas. Ya hemos apuntado que la confianza es un componente clave del capital social que, a menudo, se usa como base para medirlo. La confianza social en la sociedad tradicional china se basaba en normas éticas internas, era una confianza interpersonal. En la sociedad moderna la confianza se basa en un sistema legal externo, que se genera y existe en «organizaciones de personas jurídicas»; es, por lo tanto, una confianza institucional (Zou, Jiao y Li, 2012).

Si una nueva asociación quiere registrarse en China, debe afiliarse a alguna de las unidades comerciales que dependen del Gobierno y, para poder hacerlo, el líder o alguna persona clave en dicha asociación ha de tener guanxi con alguno de los funcionarios gubernamentales de aquella unidad comercial. Eso hará que la asociación sea aprobada. Ahora bien, en el momento que aquel líder o persona clave desaparezca, la pervivencia 
de la asociación vuelve a estar en peligro. Obviamente, este no es tanto un tema de capital social en el sentido occidental cuanto del papel que juegan el guanxi y el renqing en la vida social china. Esto supone que, en la actualidad en China, la mayoría de los recursos sociales son controlados principalmente por el personal de los departamentos gubernamentales. Además, a nivel individual, el guanxi se ha convertido en un medio para tratar con los funcionarios del Gobierno. Esto demuestra la gran importancia de la investigación sobre el guanxi para comprender la sociedad china actual. También que la teoría elaborada en occidente sobre el capital social parece no ser del todo equivalente al concepto de guanxi y que el ajuste entre ambos conceptos puede resultar problemático.

\section{El guanxi y el social capital}

El guanxi se confunde fácilmente con el capital social porque, a partir de la forma en que es definido, el guanxi es, también, una especie de red social en la que las personas tienen la oportunidad de generar, obtener y utilizar recursos sociales. Sin embargo, esta definición ignora la importancia de la tradición previa y de las influencias socioculturales del entorno. El capital social se configura a partir de personas que se involucran en relaciones sociales. En general puede decirse que los participantes en dichas relaciones suelen interesarse en las actividades colectivas y en la búsqueda del bienestar grupal o comunitario. Una vez que se establecen dichos grupos, tienen una supervisión informal y pueden servir a la economía social de la colectividad. Obviamente no nos referimos en este caso a otras formas de capital social que implican actividades delictivas o contrarias a valores cívicos.

Por el contrario, el guanxi ni tiene ese significado ni es considerado como un bien social. En principio, la mayoría de los investigadores chinos (Ke, 2018; Fei, 2015; Yanjie y Lei, 2013) tienden a considerar el funcionamiento del guanxi como la práctica y la extensión del confucianismo en la sociedad china. Esto es así porque consideran que los conceptos de benevolencia (ren), lealtad (yi), cortesía (li) y relaciones humanas (renlun) son, en el confucianismo, elementos configuradores del guanxi que han tenido un profundo impacto en la cultura social china durante miles años.

Muchos estudios (Zheng, 2011; Sang, 2011) han indicado que el guanxi se construye sobre las características de la pequeña economía campesina reflejada en la cultura agrícola china. Es decir, la familia desempeña el papel dominante en la producción y el consumo. De aquí el familiarismo al que nos hemos referido, que presupone que las familias asumirán la responsabilidad del cuidado de sus miembros en lugar del Gobier- 
no, y esto lleva a priorizar las necesidades de la familia antes que las de los individuos o las de la sociedad.

Como se ha señalado, el guanxi está muy desarrollado en la sociedad china, porque, por un lado, proviene de la influencia de los valores confucianos y, por otro, se construye sobre las relaciones territoriales y de consanguinidad propias de la sociedad rural china. No obstante, esto presenta un problema difícil de explicar, y es que el confucianismo enseña a las personas a valorar la justicia antes que el propio interés y, según este planteamiento, la familia también es una unidad viviente que debe obedecer las creencias confucianas. Por consiguiente, si exploramos el guanxi solamente desde estos dos aspectos, se hace difícil responder a porqué el guanxi tiende a ser instrumental y a buscar la rentabilidad. Esto significa que el confucianismo difícilmente sirve para para explicar, al menos al completo, las actividades y los modelos de guanxi.

A pesar de los cuestionamientos de las investigaciones sobre el guanxi, tenemos que admitir que el significado y el funcionamiento del guanxi todavía se hallan mayoritariamente restringidos por el confucianismo y la sociedad agrícola. Pero, a diferencia de lo que han señalado los autores, no creemos que el guanxi sea una extensión y una práctica del confucianismo. En general, la práctica de guanxi significa que se lleva a cabo una actividad de beneficio mutuo de forma oportunista en la red social existente. Esta forma de beneficio mutuo proviene no solo de las características de su red social, sino también del poder de la estructura social. Las características del guanxi se reflejan en parte en el concepto, ya comentado, de patrón de ondas sucesivas propuesto por Fei (2015), pero el mayor problema con este concepto es, desde nuestro punto de vista, la característica egoísta, que va en contra de la orientación de valor del confucianismo y la vida familiar.

Chang (2011) apunta que la característica instrumental del guanxi es causada por la grave escasez de recursos sociales durante el periodo de la Economía Planificada en China (desde los años 50 hasta 80 del siglo xx). Sin embargo, aunque los recursos sociales sean relativamente abundantes en la sociedad contemporánea, las prácticas del guanxi siguen siendo populares y frecuentes en China.

Algunos investigadores (Yanjie y Lei, 2013; Zheng, 2011) han dejado de explorar la conexión entre el guanxi, el confucianismo y los modos sociales chinos. Es precisamente a partir de esta ruptura cuando se comienza a usar el concepto de capital social occidental para analizar y explicar el guanxi. Un problema añadido, sin embargo, es que se está planteando el uso del guanxi de manera instrumental. Lo que significa que sería justo usar el guanxi como un atajo para perseguir los intereses personales, y esta sería una característica básica del funcionamiento normal de la sociedad.

En la vida cotidiana de la sociedad china, el guanxi se ha convertido en un principio básico. Se podría decir que hay un sentimiento encontrado (repulsión/envidia) con rela- 
ción a las personas que aprovechan el guanxi para mejorar su situación social. Desde nuestro punto de vista, se ha enfatizado en exceso la característica instrumental del guanxi, y eso ha generado una problemática persistente en la sociedad contemporánea china en relación con este tema. Eso significa que la investigación sobre el guanxi no puede simplemente adoptar, sin más, la teoría occidental del capital social.

Algunos estudiosos señalan (Li, 2013) que el guanxi obstaculiza el desarrollo del capital social en la sociedad moderna y pone en cuestión la confianza entre las personas. Sin embargo, a pesar de que haya muchas problemáticas e incorrecciones en la práctica del guanxi, esto no es algo exclusivo de China. Se podría decir que sucede algo parecido en relación con determinados usos del capital social en las sociedades occidentales.

En la Tabla 2 se pueden observar las diferencias entre el capital social y el guanxi a partir de algunas variables.

TABLA 2

Diferencias entre el capital social occidental y el guanxi chino

\begin{tabular}{|c|c|c|}
\hline & Capital social & Guanxi \\
\hline Definición & $\begin{array}{l}\text { Redes sociales generadas a partir } \\
\text { de relaciones de cooperación y } \\
\text { reciprocidad }\end{array}$ & $\begin{array}{l}\text { Redes sociales basadas en la } \\
\text { consanguinidad y en la } \\
\text { proximidad territorial y } \\
\text { geográfica }\end{array}$ \\
\hline Contexto social & $\begin{array}{l}\text { Sociedad legal y } \\
\text { constreñimientos informales de } \\
\text { tipo moral }\end{array}$ & $\begin{array}{l}\text { Sociedad regulada a través del } \\
\text { renqing }\end{array}$ \\
\hline Modo de confianza & General y abierto & $\begin{array}{l}\text { Limitado a familiares y } \\
\text { «próximos» }\end{array}$ \\
\hline Regla & Regla formal & Regla tácita \\
\hline Carácter de relación & Interés publico & Interés privado \\
\hline Efecto & $\begin{array}{l}\text { Positivo cuando es planteado } \\
\text { como bien público }\end{array}$ & $\begin{array}{l}\text { En función de cómo se usa y se } \\
\text { genera }\end{array}$ \\
\hline
\end{tabular}

Fuente: Elaboración propia.

De acuerdo con la tabla, la comprensión de las redes relacionales es bastante distinta en diferentes contextos sociales y culturales. Como señala Zhai: «La base de investigación, de la red de relaciones sociales chinas, es una sociedad basada en la familia, 
mientras que la base de investigación del capital social es la sociedad civil. Tienen diferencias significativas en la conceptualización de los individuos, la membresía, la participación, y los intereses públicos y privados» (2009: 109). Cuando los investigadores occidentales discuten sobre el capital social, lo hacen en un contexto social preestablecido regulado civil y legalmente. La movilización y el uso del capital social en las redes sociales no tiene por qué afectar a la distribución equitativa de los recursos sociales y las oportunidades sociales, por lo que parecen obedecer a la citada «hipótesis de la relación débil» (Granovetter, 1983). Por el contrario, la sociedad china afectada por tradiciones confucianas es básicamente una «sociedad caracterizada por el renqing», es decir, más por el endeudamiento entre personas que por lo que puedan decir la ley o las normas. La «red de guanxi» a menudo tiene un efecto especial que trasciende las leyes y las reglas. $\mathrm{Si}$, por ejemplo, una persona comete un delito grave y debe ser condenada a una pena muy alta según la ley, puede acabar obteniendo beneficios si dispone de guanxi con personas poderosas. Esto es algo que ocurría con mucha frecuencia en la antigüedad china. En la actualidad, la «relación fuerte» es un mecanismo operativo importante en la sociedad china. En este sistema social y cultural, la «red de guanxi» se encarna como «capital de guanxi» con interés privado.

El capital social es un concepto desarrollado en la sociedad civil occidental, producto de un pensamiento más o menos integrado sobre, entre otros, las relaciones interpersonales, la asociación, la cooperación, la confianza, la reciprocidad y la fluidez de la información (Padrò y Úcar, 2015). El guanxi, como práctica, se desarrolla a partir del funcionamiento de la sociedad familiar china, y se refiere a las relaciones con parientes, compañeros de clase social, colegas y vecinos. Es una práctica social que se fundamenta es la consanguinidad y en la proximidad territorial y geográfica.

Al comparar las diferencias operativas entre el capital social y el guanxi, la mayor diferencia es que el intercambio de información, las acciones coordinadas y las decisiones colectivas requeridas por el capital social tienen poco que ver con lo que sucede en el guanxi. En el funcionamiento del guanxi, la información no se comparte, sino que se controla. Es, de hecho, un criterio para distinguir si tienes guanxi o no. En otras palabras, comparto los recursos contigo si tienes guanxi conmigo. Hay que añadir, además, que la confianza se da a menudo, como se ha comentado, solo entre familiares.

Con el desarrollo de la moderna economía de mercado en China, el concepto tradicional de «énfasis en la justicia sobre los intereses» está cambiando gradualmente las interacciones de las personas, que parecen ser cada vez más utilitaristas. En realidad, el capital social tiene en China un gran stock, pero, en su mayoría, es capital social tradicional basado en el guanxi. Un capital social más racional, en tanto que orientado al interés público y basado en la cooperación, todavía no está ampliamente formado en la 
actualidad de la sociedad China. Se podría decir que, en general, al guanxi se le ha dado un significado especial en la sociedad actual y se ha convertido en un medio para buscar recursos sociales y obtener beneficios especiales.

El periodo de ajuste, de transición de la estructura socialista tradicional de interés a la economía de mercado socialista moderno, está generando en China numerosas problemáticas derivadas de los profundos cambios que supone en todos los estamentos y niveles relacionales entre personas, administraciones y organizaciones. Un ejemplo lo constituye el sistema legal. Es difícil obtener respuesta a las demandas personales a través de canales normales o participar en el mercado y en la asignación de recursos de manera abierta y en condiciones equitativas, por lo que, una manera de sortear estos obstáculos es recurrir al guanxi. Desde nuestro punto de vista, este tipo de guanxi corresponde a un capital social negativo. En sentido positivo, el guanxi ayuda a identificar valores dentro de los grupos sociales, a reducir los costos de transacción y a mejorar la eficiencia social. En un sentido negativo, aumenta el cierre social y no favorece la apertura de recursos y la igualdad de oportunidades.

Como la economía de mercado está necesariamente sujeta a una regulación legal, especialmente en lo que respecta a los derechos de propiedad y el contrato, la integración más completa de China en la economía internacional globalizada requerirá un desarrollo institucional que, posiblemente, pueda acabar desplazando al guanxi. Chang (2011), que desarrolla un enfoque evolutivo, argumenta que la «forma de acceso» del guanxi, que se utiliza para adquirir algo deseable, está disminuyendo en China, mientras que una «forma de inclusión» de guanxi, que enfatiza el cultivo de la confianza y la comprensión en conexiones mutuamente beneficiosas, está ganando mayor importancia.

\section{CONCLUSIONES}

En la última década se ha despertado un interés creciente, en el ámbito de las ciencias sociales, en relación con una práctica social que parece estructurar las relaciones sociales en la cultura China; el guanxi. Una práctica que media las relaciones interpersonales, comerciales, políticas y sociales que, en principio, resulta extraña para la cultura occidental.

Los investigadores occidentales han intentado sortear la brecha cultural a través de conceptos y prácticas propios que les ayuden a comprender una práctica tan diferente. El concepto con el que se ha intentado equiparar el guanxi ha sido el de capital social. La razón principal por la que se ha buscado este paralelismo es porque ambos, guanxi y capital social, suponen la creación de una red de relaciones que se construyen y susten- 
tan, fundamentalmente, por la confianza. Sin embargo, a menudo se ha aplicado la teoría occidental sobre el capital social al estudio del guanxi ignorando o minimizando las diferencias socioculturales entre ambos contextos.

Este artículo realiza una revisión de la literatura académica que analiza las relaciones entre el guanxi y el capital social en la última década. En él se definen, analizan y ponen en relación los conceptos de guanxi, capital social, renqing, dimensiones culturales y filosofía antigua china.

Se ha caracterizado el guanxi como un vínculo relacional que obliga a las personas a proporcionar favores (renquing) a sus familiares y a las personas con las que han establecido este tipo de vínculo, y a esperar, en consecuencia, la reciprocidad de dichas personas en el futuro.

La mayor parte de la práctica del guanxi, descrita en la literatura existente, involucra costumbres y rituales tradicionales chinos que parecen vincularse mucho con el parentesco, la proximidad territorial y los valores propios del confucianismo. En el análisis se han identificado tres perspectivas: la «ego-práctica», que pone el foco en la mejora del propio individuo; la «ética comunitaria», que enfatiza que el guanxi beneficia solo a determinados individuos y cuestiona la justicia social y la equidad; y, por último, la «relación confuciana», que supone que hay una orientación natural hacia el guanxi que se construye a partir del parentesco, la familiaridad y el grado de conocimiento y cercanía. A partir de esta última perspectiva se ha hablado también de tres tipos de guanxi: el que se produce en una relación familiar; en una relación con personas conocidas; y, por último, en una relación con personas desconocidas.

La mayoría de las definiciones elaboradas en Occidente en relación con el capital social coinciden en caracterizarlo como un conjunto de recursos disponibles para los individuos que son adquiridos a través de su participación en redes sociales. De las tres perspectivas esenciales que lo caracterizan, la estructural, la relacional y la orientada a los recursos, parece ser esta última la que ofrece una mejor definición del nivel individual de capital social en un contexto guanxi. También se ha podido establecer un paralelismo entre los tipos de guanxi definidos y los tipos de capital social establecidos por Putnam, bonding, bridging y linking.

La dificultad para comprender las relaciones entre el capital social occidental y el capital social chino se derivan de las transformaciones operadas en la sociedad china por el paso de una sociedad agrícola a una industrial y tecnológica y por los cambios que han supuesto en relación con los valores tradicionales y la manera de entender las relaciones. En la actualidad parece haber una clara orientación al mantenimiento de la gran mayoría de asuntos dentro del entorno familiar y al establecimiento generalizado de normas sociales uniformes para todas las personas. Esa es la razón por la que el guanxi 
se ha convertido en un importante instrumento para tratar con el funcionariado gubernamental. Es por eso que se habla de un uso instrumental del guanxi, que se contrapone a los valores clásicos del confucianismo, y de los obstáculos que puede suponer para el desarrollo del capital social en el sentido que tiene en Occidente.

El guanxi es una red, y eso significa que puede ser interpretado como uno de los componentes del capital social más que como capital social propiamente dicho. Es muy probable que el guanxi siga desempeñando un papel en la vida social y económica de China. Ciertamente, como las formas legales tienden gradualmente a predominar, los usos del guanxi en prácticas corruptas y el soborno van, sin duda, a disminuir. Sin embargo, parece altamente probable que la importancia del guanxi para ganar y mantener la confianza, proporcionar ventajas en los costos de transacción y ofrecer apoyo mutuo a quienes comparten relaciones recíprocas siga existiendo.

Explorar la relación entre guanxi y el capital social ayuda a profundizar la comprensión de la relación social entre los países occidentales y China. En un contexto de intercambios internacionales cada vez más frecuentes se hace necesario comprender la cultura si se busca promover la cooperación entre las dos partes.

A pesar de que existen divergencias entre el guanxi y el social capital, no se puede negar que ambos conceptos pueden influirse entre sí, dadas, por una parte, las similitudes que comparten y, por otra, la globalización de la investigación de la investigación académica que favorece la intercomunicación y el contraste de ideas y conceptos. Se podría decir, por ejemplo, la inversión en la construcción social y económica a través de gremios y asociaciones es una transformación de guanxi en capital social. El tratamiento preferencial de familiares y amigos en la red a través de proyectos nacionales es una transformación de capital social en guanxi.

\section{REFERENCIAS BIBLIOGRÁFICAS}

Barbalet, J. (2018). Guanxi as social exchange: Emotions, power and corruption. Sociology, 52(5), 934-949. https://doi.org/10.1177/0038038517692511

Bourdieu, P. (1986). The Forms of Capital. En J. Richardson (Ed.), Handbook of theory and research for the sociology of education (241-258). Nueva York, EE. UU.: Greenwood Press.

Chang, K. C. (2011). A path to understanding guanxi in China's transitional economy: Variations on network behavior. Sociological Theory, 29(4), 315-339. https://doi. org/10.1111/j.1467-9558.2011.01401.x 
Chen, Y., Friedman, R., Yu, E., y Sun, F. (2011). Examining the positive and negative effects of guanxi practices: A multi-level analysis of guanxi practices and procedural justice perceptions. Asia Pacific Journal of Management, 28(4), 715-735. https:// doi.org/10.1007/s10490-009-9176-x

Coleman, J. S. (1988). Social Capital Capital socialin the Creation of Human Capital. The American Journal of Sociology, 94, 95-120. https://doi.org/10.1086/228943

Confucius. (2013). The book of rites (li Ji). English-Chinese Version. Beijing/Washington, DC: Intercultural Press.

Fei Xiaotong. (2015). Patrón diferencial. Wen Yuan: Literatura clásica (6), 46-47.(Traducido de 费孝通. 2015. 差序格局. 文苑: 经典美文, (6), 46-47.)

Granovetter, M. 1983. The strength of weak ties: A network theory revisited. Sociological theory, 201-233. https://doi.org/10.2307/202051

Huang, W. Y., Huang, C. Y., y Dubinsky, A. J. (2014). The impact of guanxi on ethical perceptions: The case of Taiwanese salespeople. Journal of business-to-business marketing, 21(1), 1-17. https://doi.org/10.1080/1051712X.2014.857499

Ip, P. K. (2008). Corporate social responsibility and crony capitalism in Taiwan. Journal of business ethics, 79(1-2), 167-177. https://doi.org/10.1007/s10551-007-9385-5

Jia, L., You, S. y Du, Y. (2012). Chinese context and theoretical contributions to management and organization research: A three-decade review. Management and Organization Review, 8(1), 173-209. https://doi.org/10.1111/j.1740-8784.2011.00282.x

Ke, X. (2018). Succession and the Transfer of Social Capital Capital socialin Chinese Family Businesses: Understanding Guanxi as a Resource-Cases, Examples and Firm Owners in Their Own Words (Vol. 25). V\&R unipress GmbH. https://doi. org/10.14220/9783737008921

Khatri, N., Tsang, E. W., y Begley, T. M. (2006). Cronyism: A cross-cultural analysis. Journal of International Business Studies, 37(1), 61-75. https://doi.org/10.1057/palgrave.jibs. 8400171

Li D. (2013). «Investigación sobre el papel del capital social en el campo de la filosofía política». Tesis doctoral, Departamento de filosofía, Universidad de Heilongjiang (Traducido de 李东旭. 2013. 政治哲学视域的社会资本作用研究. Doctoral dissertation, 黑龙江大学)

Li, S. X., Yao, X., Sue-Chan, C., y Xi, Y. (2011). Where do social ties come from: Institutional framework and governmental tie distribution among Chinese managers. Management and Organization Review, 7(1), 97-124. https://doi. org/10.1111/j.1740-8784.2010.00187.x 
Liu, L., y Mei, S. (2015). How can an indigenous concept enter the international academic circle: the case of guanxi. Scientometrics, 105, 645-663 https://doi.org/10.1007/ s11192-015-1687-2

Luo, Y. 2019. International investment strategies in the People's Republic of China. Routledge. https://doi.org/10.4324/9780429431777

Luo, Y., Huang, Y., y Wang, S. L. (2012). Guanxi and organizational performance: A meta-analysis. Management and Organization Review, 8(1), 139-172. https://doi. org/10.1111/j.1740-8784.2011.00273.x

Mao, Y., Peng, K.Z. \& Wong, CS.(2012). Indigenous research on Asia: In search of the emic components of guanxi . Asia Pac J Manag 29, 1143-1168 https://doi. org/10.1007/s10490-012-9317-5

Moher D, Liberati A, Tetzlaff J, Altman DG and The PRISMA Group. (2009). Preferred Reporting Items for Systematic Reviews and Meta-Analyses: The PRISMA Statement. PLoS Med 6(7): e1000097. https://doi.org/10.1371/journal.pmed.1000097

Padró, M. y Úcar, X. (2015). Comunidades en movimiento: los planes de desarrollo comunitario como promotores de capital social. Educación Social. Revista d'Intervenció Socioeducativa (59), 115-130

Peck, J., y Zhang, J. (2013). A variety of capitalism... with Chinese characteristics? Journal of Economic Geography, 13(3), 357-396. https://doi.org/10.1093/jeg/lbs058

Putnam, R. (1993). The Prosperous Community: The Social Capital Capital social and Public Life. The American Prospect. 4 (13). Pp. 35-42

-(1995). «Bowling alone: America'sdeclining social capital». Journal of Democracy, $6,65-78$

Qi, X. (2012). A case study of globalized knowledge flow: Guanxi in social science and management theory. International Sociology, 27 (6), 707-723. https://doi. org/10.1177/0268580912453729

- (2013). Guanxi, social capital capital socialtheory and beyond: Toward a globalized social science. The British journal of sociology, 64(2), 308-324. https://doi. org/10.1111/1468-4446.12019

Sang L. (2011). El dilema y la vergüenza de la transformación de la modernidad de los recursos culturales tradicionales chinos. Tomando Ninigou en Huaiyang en Henan como ejemplo. Decoración, (2), 122-124. (Traducido de 桑林. 2011. 中国传统文 化资源现代性转型的困境与塩近一 - 以河南淮阳泥泥狗为例. 装饰, (2), 122124.)

Sennett, R. (2012). Juntos. Rituales, placers y política de cooperación. Barcelona: Anagrama. 
Velez-Calle, A., Robledo-Ardila, C., \& Rodriguez-Rios, J. D. (2015). On the influence of interpersonal relations on business practices in Latin America: A comparison with the Chinese guanxi and the Arab Wasta. Thunderbird International Business Review, 57(4), 281-293.

Wang S. y Kang Z. (2012). El «capital cuasi-social» de la sociedad conocida china: un estudio sobre las reservas de social capital capital socialde la sociedad china tradicional. Frente de ciencias sociales, 1. (Traducido de 王尚银, \& 康志亮. 2012. 中国 熟人社会的《类社会资本》一关于中国传统社会社会资本储量的考究. 社会 科学战线, 1.)

Wang, P. (2014). Extra-legal protection in China: How guanxi distorts China's legal system and facilitates the rise of unlawful protectors. British Journal of Criminology, 54(5), 809-830. https://doi.org/10.1108/S1479-8387(2012)0000008003

Wijaya, Y. (2008). The prospect of familism in the global era: A study on the recent development of the ethnic-Chinese business, with particular attention to the Indonesian context. Journal of business ethics, 79(3), 311-317. https://doi.org/10.1007/ s10551-007-9399-z

Woolcock, M. (2001). The place of social capital in understanding social and economi coutcome.https://www.researchgate.net/publication/200031275_The_Place_of_Social_Capital_in_Understanding_Social_and_Economic_Outcomes (descargado, enero, 2011).

Xiaoxin, W. (2013). The power of social capital capital socialin school choice in a Chinese city. Australian Journal of Education, 57(1), 48-59. https://doi. org/10.1177/0004944112468701

Yanjie, B., Lei, Z. (2013). Sobre cultura guanxi y social capital capital socialguanxi. Journal of Humanities, 1, 107-113. (Traducido de 边燕杰, \& 张磊. 2013. 论关系文 化与关系社会资本. 人文杂志， 1， 107-113.) Doi:10.3969/j.issn.0447-662X.2013.01.014

Zhai X. (2009). ¿Es "guanxi" o social capital? Sociedad, 29 (1), 109-121. (Traducido de 翟学伟. (2009). 是 《关系》, 还是社会资本. 社会, 29(1), 109-121.)

Zhang, L., Deng, Y. y Wang, Q. (2014). An exploratory study of Chinese motives for building supervisor-subordinate guanxi. Journal of Business Ethics, 124(4), 659675. https://doi.org/10.1007/s10551-013-1899-4

Zhang, N. (2011). The impact of guanxi networks on the employment relations of rural migrant women in contemporary China. Industrial Relations Journal, 42(6), 580595. 
Zheng A. (2011). Sobre la cultura tradicional de China y la formación de social capital. Economía contemporánea, 7, 24-25. (Traducido de 郑艾林. 2011. 论我国传统文 化与社会资本形成. 当代经济, 7, 24-25.)

Zou, X., Cheng, Y., \& Nie, J. B. (2018). The social practice of medical guanxi (personal connections) and patient-physician trust in China: an anthropological and ethical study. Developing world bioethics, 18(1), 45-55.

Zou, Y, Jiao, D y Li J. (2012). El patrón de confianza y el impacto del social capital capital socialde los residentes urbanos chinos. Chinese Social Sciences, 5, 131-148. (Traducido de 邹宇春, 教丹, \& 李建栋. 2012. 中国城市居民的信任格局及社会 资本影响. 中国社会科学, 5, 131-148.) 